\title{
Postprint
}

\section{Politische Deliberation online - Twitter als Element des politischen Diskurses}

\author{
Caja Thimm, Jessica Einspänner, Mark Dang-Anh
}

\section{Einleitung}

Die These, dass das Internet die Strukturen von Kommunikation verändert, ist ein zentrales Paradigma der Debatte um die Mediatisierung der Gesellschaft (Krotz 2007). Insbesondere der öffentliche Diskurs befindet sich in einem grundlegenden Wandel, da Öffentlichkeit nicht mehr allein von etablierten, professionellen Medienschaffenden, sondern zunehmend von institutionell nicht privilegierten Individuen gestaltet wird, die ihre eigene Umwelt zum Gegenstand des öffentlichen Diskurses machen. In der mediatisierten Gesellschaft wird Öffentlichkeit so ein gemeinsamer politischer (Ver-)Handlungsraum, in dem das Internet immer wichtiger für kollektiv bindende Entscheidungen wird. Konkret für die Politik ist es sogar wichtiger als andere Faktoren wie Parteiapparate, Interessengruppen, Experten oder internationale Beziehungen (Vowe 2006: 441).

Bezüglich dieser Veränderungen zeichnen sich zwei Sichtweisen ab, die in ihren Bewertungen kategorial differieren. Einerseits finden sich explizit medienenthusiastische bzw. cyberoptimistische Positionen, die von neuen Optionen der Partizipation ausgehen (Plake/Jansen/Schumacher 2001) oder ein globales Kaffeehaus im Entstehen sehen (Grunwald/Banse/Coenen/Hennen 2006). Hier wird der Computer als ein Medium angesehen, mit dem frühe Mündlichkeitskulturen technisch wiederherstellbar sind. Elektronische Gemeinschaften werden in dieser Konzeption als Substrate längst verloren gegangener öffentlicher Orte der Kontaktaufnahme und Kommunikation gewertet, als öffentliche Kommunikationsformen in der Tradition der Salons und Kaffeehäuser des 18. Jahrhunderts eingeordnet und als „elektronische Agora“ (Lévy 1998, 2001) beschrieben. Einher geht damit die Vorstellung, dass eine kommunikative Kultur, wie sie vor der Einführung der Schrift in traditionellen Mündlichkeitskulturen existierte, technisch wiederherstellbar sei. Damit wird das Netz als Ort der technischen Verwirklichung der Moderne 
verstanden, als Forum idealisierter Kommunikationskulturen, in dem die Weisheit der Masse (Surowiecki 2004) die besten Ideen für die Gesellschaft produziert.

Die medienkritische (oder cyberpessimistische) Perspektive dagegen ist von grundlegender Skepsis gegenüber überhöhten Erwartungen an das Netz gekennzeichnet und geht davon aus, dass durch die computervermittelte Kommunikation Verluste an Sozialität, Intimität und intellektueller Kompetenz zu verzeichnen sind (Thurlow/Lengel/Tomic 2004). Zudem wird konstatiert, dass das Internet ein Medium der Ungleichheit sei und eine digitale Spaltung befördere (Marr/Zillien 2010; Norris 2001). Besonders gegen die Vorstellung vom Kollektivcharakter des Internets mehren sich kritische Stimmen, so Keen mit seiner These vom „Kult der Amateure“ (2007) und Lanier in seiner Streitschrift zum „Digital Maoism“ (2006).

Betrachtet man unter diesen Perspektiven die Mediatisierung des Politischen, zeigen sich die Hoffnungen und Befürchtungen besonders deutlich. Die optimistische Perspektive ist hier mit der Idee verbunden, nach der das Internet ein Ort deliberativer Demokratie sein kann, an dem sich Politik und Zivilgesellschaft konstruktiv über politische Entscheidungen austauschen und diese so legitimieren. Die übergeordnete Fragestellung, welche das Habermas'sche Kommunikationsideal als Ausgangskonzept sieht (Habermas 1962/1990), beruft sich auf die positiven Aspekte von erhöhten Partizipationsoptionen und globaler Vernetzung der Bürgerschaft.

Die vorliegende Studie versucht einen Beitrag zur Erforschung der Mediatisierung des Politischen zu leisten und untersucht, ob der Microbloggingdienst Twitter eine Plattform für deliberativen Meinungsaustausch darstellen kann. Grundlage der empirischen Ausführungen sind umfangreiche Daten zur TwitterKommunikation von PolitikerInnen und Bürgerschaft im kommunikativen Kontext von Landtagswahlkämpfen.

Als Ausgangspunkt für die Überlegungen ist jedoch zunächst zu fragen, ob und inwieweit sich das Habermas'sche Modell des deliberativen Diskurses auf die digital mediatisierten Bedingungen im Netz übertragen lässt. Die unterschiedlichen Kommunikationsbedingungen von Netzdiskursen bilden den Hintergrund für die Frage nach der ,digitalen Deliberation“.

\section{Digitale Deliberation}

Der Netzwerkgedanke des klassischen Deliberationsmodells wird seit einigen Jahren von der Onlineforschung aufgegriffen und für die Internetkommunikation adaptiert. Ausschlaggebend für diese Adaption ist die Kategorie des Diskurses: 
Kennzeichnend für die deliberative Demokratietheorie ist vor allem die inputorientierte Legitimation politischer Entscheidungen durch einen gemeinschaftlichen Diskurs, durch den sich bei den Beteiligten politische Präferenzen formen und herausbilden. Dieser Diskurs bildet sich im Netz öffentlich ab, so eine der Thesen.

\subsection{Grundlagen deliberativer Demokratiemodelle}

Im Fokus des klassischen Modells stehen die kommunikativen Praktiken der Diskursteilnehmer, in denen politische Fragen in institutionellen oder informellen Diskursnetzwerken ausgehandelt werden (Habermas 1996: 285). Deliberationen (Beratschlagungen, Überlegungen) in institutionellen Netzwerken beziehen sich sowohl auf den politischen Input als auch auf den Output. Damit sind etwa Verfahren von Aushandlungen politischer Fragen in Bürgerversammlungen, Mediationsprozessen oder Deliberationsforen (,deliberative polls“, Fishkin 1991) gemeint, an deren Ende ein kollektiv verbindlicher Entschluss steht. Die kommunikativen Diskurspraktiken konstituieren sich hier durch festgelegte Regeln, die jedoch selbst auch thematisiert und geändert werden können. Im Gegensatz dazu vollziehen sich Deliberationen in informellen Netzwerken ausschließlich auf der Input-Seite politischer Prozesse. Sie haben nicht den Charakter zeitlich abgrenzbarer, abgeschlossener (Dezisions-) Verfahren, sondern können im Laufe der Zeit Meinungs- und Willensbildungen mit unterschiedlichen Themenschwerpunkten entwickeln. Deliberative Verfahren in informellen Netzwerken sind demnach ungeregelt und diskontinuierlich, die Zusammensetzung ihrer Teilnehmer ist dispers. Dennoch unterliegen sie spezifischen Bedingungen, die in der Beschaffenheit des jeweiligen Mediums begründet liegen. Informelle Diskursnetzwerke können weitreichend und die Zusammensetzung ihrer Partizipienten kann sehr heterogen sein, was aus Deliberationsperspektive als Stärke zu sehen ist: „The virtues of the public sphere, where deliberation takes place, originate from the fact that a group of people with different opinions and different backgrounds engage in a public debate" (Witschge 2004: 110).

War für Habermas noch das gemeinsam erarbeitete und im Konsens entschiedene Ergebnis der Deliberation zentral, so hat sich diese Sichtweise in der aktuellen Forschung modifiziert. Die Vielfältigkeit der Meinungen formiert sich vielmehr (auch) im Dissens: „Deliberative Theory has moved away from a consensus-centered teleology - contestation and indeed the agonistic side of democracy now have their place“ (Chambers 2003: 321). Das ist jedoch nicht gleichbedeutend mit der Verabschiedung von der Annahme vernunftgeleiteter 
Beratungen, denn ,reason-based collective formation and consensus are not the same thing" (Fishkin 2009).

Abgesehen von den Unterscheidungen zwischen institutionellen und informellen Netzwerken oder dissens- und konsenszentrierten Debatten, sind es jedoch vor allem die spezifischen formalen Bedingungen deliberativer Diskurse, die das deliberative Demokratiemodell auszeichnen. Habermas sieht folgende Voraussetzungen als normativ für den deliberativen Diskurs an: Egalität aller Diskursteilnehmer (einschließlich des Publikums), prinzipielle Problematisierbarkeit aller Themen und Meinungen und Einhalten der diskursethischen Kriterien (Habermas 1983). Zusammengefasst bedeutet dies, dass jeder Bürger/jede Bürgerin an der öffentlichen politischen Diskussion teilhaben und sich mit seiner eigenen Meinung einbringen kann und soll. Dabei sollen Regeln gelten, die für alle vertretbar scheinen und schließlich zu einem Konsens führen. Deliberative Demokratieprozesse lassen sich also als Prozesse politischer Meinungs- und Willensbildung (Input) der Bürger einer Demokratie beschreiben, die sich rational in öffentlichen Diskursen vollzieht und kollektiv verbindliche Entscheidungen des politischen Systems (Output) legitimiert. Deliberative Diskurse konstituieren sich dabei durch gleichwertige Diskursbeiträge zu einem bestimmten Themenbereich, die aufeinander Bezug nehmen und sich in Referenzierungs- und Sprechhandlungen ausdifferenzieren.

\subsection{Perspektiven auf Online-Deliberation}

Die genannten Kriterien sind nicht nur für die klassische Deliberationstheorie kennzeichnend, sondern werden auch für die gegenwärtigen Modellierungen von Online-Deliberation herangezogen. Hierbei stellt sich die Frage, inwiefern sich die etablierten Konzepte und Vorstellungen über die deliberativen Anteile demokratischer Prozesse auf die Netzkommunikation übertragen lassen.

Die Herangehensweise an den politischen Diskurs im Online-Medium ist, wie auch die Perspektiven auf das Netz insgesamt, durch polarisierte Haltungen geprägt. Auf der einen Seite sehen die sogenannten Cyberenthusiasten im Internet grundsätzlich eine vielversprechende Technologie, die zur Verbesserung der Demokratie durch mehr Partizipations- und Interaktionsmöglichkeiten führt (Dyson 1997; Negroponte 1995; Rheingold 1993). Die Möglichkeiten eines schnellen, kostengünstigen Zugriffs auf politische Informationen und Teilnahme an politischen Prozessen sowie die einfache, ungehemmte Kommunikation (Döring 2008; Kiesler/Siegel/McGuire 1984) zwischen den Menschen im Internet kommen den Idealvorstellungen des deliberativen Demokratiemodells sehr nahe. Die 
Netzoptimisten vertreten die Ansicht, dass die digitalen InteraktantInnen egalitär die eigene Meinung zu einem gewählten, politisch relevanten Thema kundgeben und damit eine Öffentlichkeit im Habermas'schen Idealmodell konstituieren: als „Sphäre der veröffentlichten Meinungen“ (Habermas 2008: 166, Hervorhebung im Original), in der die individuellen Onlinediskursinhalte Symptome politischer Deliberation sind. Hierunter zählt auch die Vorstellung, dass das Internet vor allem ein informelles Forum für Bürger sein soll, d. h. „staatliche Interventionen auf ein Minimum beschränkt" bleiben (Winkel 2001: 144).

Die Hoffnungen der Cyberenthusiasten konnten empirisch bisher kaum belegt werden. Die Erwartungen an eine kritische, rational argumentierende und tolerante Internetöffentlichkeit, die digitale Räume politischer Deliberation erschließt, scheinen zu anspruchsvoll. Damit sehen sich die Cyberpessimisten in ihrer Grundannahme bestätigt, die auf der anderen Seite der Kontroverse bessere demokratische Diskurse durch den Einfluss des Internets negieren. So wird im Hinblick auf die vermeintlich positive Meinungsvielfalt und zahlreichen Möglichkeiten der intellektuellen Reflexion u. a. damit argumentiert, dass Menschen im Internet gezielt ihren Präferenzen entsprechende Inhalte rezipieren und sich gerade nicht über konträre Standpunkte informieren (Sunstein 2001: 49; Winkel 2001: 150). Die Folge, so die These, sei eine Polarisierung und Segmentierung der Internetöffentlichkeit, sodass sich die Menschen immer mehr von einer (ohnehin niemals erreichten) idealen öffentlichen Hauptarena entfernen und beginnen, sich in ihren eigenen Informationswelten sozial zu isolieren (Witschge 2004). Dies führt gemäß der cyberpessimistischen Perspektive zu einem starken partizipatorischen Ungleichgewicht, bei dem die ohnehin politisch aktiven Gruppen zu einer digitalen Informationselite heranwachsen, die sich stark politisch engagiert, während andere teilnahmslos und ,apathisch“ abseits stehen (Norris 2001: 98). Das verheißene deliberative Potenzial wird dem Internet durch die sogenannte Fragmentierungsthese aberkannt: Es bilden sich demnach zunehmend divergente Teilöffentlichkeiten, „so dass der Gesellschaft die gemeinsame Basis für den politischen Diskurs abhanden kommt, eine kollektive Meinungs- und Willensbildung erschwert wird“" (Schulz 2011: 130). Auch Morozov (2009) stellt die Macht des Netzes als grundsätzlich demokratiefördernd in Frage. Das Netz könne einerseits autoritäre Systeme angreifen und Demokratieentwicklungen begünstigen, andererseits als ein einflussreiches Werkzeug fungieren, um autoritäre und nationalistische Anschauungen überhaupt erst zu verbreiten und Regimen somit in die Hände zu spielen.

Wenngleich beide Sichten auf das Demokratie- und Partizipationspozential des Internets ,teilweise übersteigert“ (Perlot 2008: 86) wirken, müssen selbst Öffentlichkeitstheoretiker wie Jürgen Habermas abwägen: „Der begrüßenswerte 
Zuwachs an Egalitarismus, den uns das Internet beschert, wird mit der Dezentrierung der Zugänge zu unredigierten Beiträgen bezahlt. In diesem Medium verlieren die Beiträge von Intellektuellen die Kraft, einen Fokus zu bilden" (Habermas 2008: 82). Es finden sich zunehmend Argumente gegen das Festhalten an den Extrempositionen oder einem ,unidirektionalen Kausaleffekt entweder in Richtung mehr, weniger oder gleichwenig Demokratie" (Yang 2008: 2) des Internets. Vielmehr wird es ,,als sozial wandelbares Medium begriffen, das in unterschiedlichen Teilbereichen der Öffentlichkeit unterschiedliche Effekte und zwar in Abhängigkeit der jeweils sozial gegebenen Situationsdefinition zeitigt" (ebenda).

Es ist aber ebendiese situative Bedingtheit, die bisher zu wenig Berücksichtigung erfahren hat. Die hohe Ausdifferenzierung in Netzkulturen mit unterschiedlichen technologischen Hintergründen, Aktivitätsoptionen und Beteiligungsformen muss jeweils spezifisch analysiert und beschrieben werden.

\subsection{Demokratie und Social Web}

Den Gegenstand aktueller Forschung zum Demokratiebeitrag des Internets stellen vor allem die neuen Onlineformate wie soziale Netzwerke oder Blogs und Microblogs dar (z. B. Bessette/Pitney 2011; Haas Lyons 2011; Petrik 2010). Dabei scheint es, als wiederholten sich die frühen Kontroversen der Netzoptimisten und -pessimisten teilweise (Thierer 2010; Thimm 2008) und übersetzten sich bis in einzelne Applikationen. Nun geht es jedoch nicht mehr um die generelle Frage, ob das Internet Menschen zu mehr politischer Aktivität mobilisieren kann, sondern ob beispielsweise Facebook, Twitter oder Weblogs durch das Engagement vieler Partizipierender politische Ereignisse beeinflussen oder gar zum Wandel politischer Systeme führen, wie dies beim sogenannten „Arabischen Frühling“ 2011 gemutmaßt wurde.

Im Mittelpunkt dieser Debatte steht die Funktionalität der Mobilisierung und der Informationsdiffusion durch die schnellen und mobilen Endgeräte. Für genau diese Funktionen lassen sich eine Vielzahl von Beispielen finden. Immer häufiger werden soziale Netzwerke z. B. von Unterstützern regimekritischer Gefangener genutzt, um auf diese Weise Druck auf die Regierung auszuüben (wie es z. B. bei dem chinesischen Regimekritiker Liu Xiaobo und dem Microbloggingdienst Sina Weibo Ende 2010 der Fall war) oder auch, um in sogenannten „Safer Blogger Organisationen“" (z. B. Committee to Protect Bloggers, Electronic Frontier Foundation, Open Net Initiative) für deren Freilassung zu kämpfen. Die Macht der digitalen Koordinationsfähigkeiten lässt sich auch an Beispielen ablesen, die erst im Verlauf des Geschehnisses politische Relevanz erhalten, wie im Fall der 
Plagiatsaffäre des ehemaligen deutschen Verteidigungsministers zu Guttenberg im Februar 2011. In dessen Doktorarbeit wurden durch die akribische Prüfung vieler Freiwilliger auf einem Wiki (http://de.guttenplag.wikia.com) zahlreiche nicht kenntlich gemachte Zitate nachgewiesen, wodurch zunächst im Social Web und daraufhin in der breiten Öffentlichkeit eine Kontroverse um die Glaubwürdigkeit des Ministers ausgelöst wurde, die letztlich in seinem Rücktritt endete. Der Erfolg dieser Aktivität führte zu einer Ausweitung der Recherchen auf die Dissertationen weiterer PolitikerInnen, die zum Entzug des Titels bei mehreren der Untersuchten führte (siehe z. B. http://de.vroniplag.wikia.com).

Während also auf der einen Seite dem Social Web als politisches Verlautbarungstool für seine NutzerInnen eine stetig wachsende Einflusskraft zugesprochen wird (z. B. Boyd 2008; Idle/Nunns 2011; Shirky 2009), stehen auf der anderen Seite viele der vermeintlich revolutionären Kraft von Anwendungen wie Twitter äußerst skeptisch gegenüber (Heacock 2009; Morozov 2009). Sie bestreiten zwar nicht, dass Bürgerinnen und Bürger sich mit Hilfe sozialer Medien organisieren können, verweisen aber darauf, dass auch autokratische Autoritäten soziale Netzwerke anzuwenden wissen: „Repressive governments are becoming better at using these tools to suppress dissent" (Shirky 2011). Ferner, so wird argumentiert, sei der vermeintlich starke Einfluss digitaler Kommunikate auf das politische Geschehen hauptsächlich von den Massenmedien hochstilisiert worden: „The Twitter Revolution seems to be largely a product of the media” (Heacock 2009).

Neben der unbestreitbaren Tatsache, dass Medien immer schon zur Verbreitung politischer Inhalte genutzt wurden, lässt sich jedoch der im Zuge der Mediatisierung der Gesellschaft beobachtbare Grad an Partizipation, d. h. die Ausweitung der am Diskurs beteiligten Gruppierungen, durchaus als eine kategoriale Veränderung begreifen. Sieht man die digitale Revolution auf derselben Ebene wie die Revolution der Alphabetisierung im Zuge der Technikrevolution des Buchdrucks, so wird deutlich, dass nur die Verschränkung von Technologie (des Gerätes) und Technologiekompetenz (der Nutzer) die gesellschaftlichen Umbrüche in der Folge der Mediatisierung erklären können.

\subsection{Aspekte der Online-Deliberation}

Bei der Bestimmung und Analyse von Online-Deliberation bereitet vor allem die Tatsache Schwierigkeiten, dass bisher nur wenige empirische Ansätze eines direkten Vergleichs zu Offline-Deliberation vorliegen. Gut begründen lässt sich zunächst, dass digitale soziale Netzwerke für den Meinungsaustausch eine wichtige Rolle spielen. Bei einer Untersuchung von Themen und 
Diskussionsstrukturen parteiischer Weblogs stellen Koop/Jansen fest, dass unter den Bloggern nicht zwangsläufig Egalität herrscht. Jedoch werten die Autoren die Tatsache, dass in den untersuchten Blogs vor allem strittige Punkte diskutiert werden, als ein Element demokratischer Deliberation. Problematisch sehen sie allerdings die grundsätzliche Zugänglichkeit: „If a debate is dominated by few voices, then deliberation has not engaged the views of the community widely" (Koop/Jansen 2009: 157).

Auch Witschge (2004) und Mutz (2002) sehen kontroverse Themen als wichtige Voraussetzung zur Entwicklung deliberativ-demokratischer Diskurse, wenngleich ,exactly this difference and disagreement [...] make people avoid deliberative politics" (Witschge 2004: 119). Angespielt wird hier auf die Hypothese, dass Menschen vornehmlich mit Personen interagieren, die ähnliche Ansichten und Werte vertreten wie sie selbst. Besonders bei der teils anonymisierten Kommunikation im Internet könnte dies einen deliberativen Austausch erschweren. Bereits in der klassischen Deliberationstheorie wurde der Konsens, wie ihn Habermas normativ verankerte, als ideal und kontrafaktisch gesehen (Brosda 2010).

Aus einem Experiment mit 81 Studierenden, von denen einige online und andere offline miteinander bestimmte Themen deliberativ diskutieren sollten und anschließend darüber befragt wurden, schlussfolgert Min (2007): „The results support the hypotheses that both face-to-face and online deliberation will have positive impacts on the participants' issue knowledge, political efficacy, and willingness to participate in politics“. Ferner stellt sie fest, dass Deliberation in der Onlineumgebung gegenüber Face-to-Face-Deliberation einige Vorteile mit sich bringt, zumal eine größere Anzahl von DiskutantInnen - unabhängig von geografischen Limitationen - teilnehmen kann.

Habermas geht in seinen Überlegungen zur Bestimmung von Deliberation von den Grundsätzen Zugänglichkeit, Chancengleichheit, Rationalität, Informiertheit und Kontroversität aus. Eine einfache Übertragung dieser Aspekte in die Online-Umgebung erscheint problematisch, da der Kontext der Netzkommunikation zu berücksichtigen ist. So lässt sich beispielsweise die von Habermas postulierte prinzipielle Zugangsfreiheit zum Diskurs für potentielle TeilnehmerInnen nicht grundsätzlich voraussetzen, da nach wie vor Beschränkungen in vielen Ländern und sozialen Gruppen für Ausschlussprozesse verantwortlich sind. Auch die Bedingung der Chancengleichheit der Beteiligung (Partizipierende müssen egalitäre Diskurspartner sein) erscheint nur bedingt übertragbar.

Als Beschreibungsaspekte deliberativer Prozesse, die sich dagegen gut im Netz abbilden lassen, können die Kriterien Rationalität, Informiertheit und Kontroversität angesehen werden. Rationale, also begründete und argumentationsgeleitete Diskussionen durch Diskussionsteilnehmer, die die Möglichkeit haben, 
sich zum jeweiligen Thema (Hintergrund-)Informationen einholen zu können, um ihre Argumentationen zu stützen, sowie die politische Relevanz und Kontroversität der Themen, erscheinen daher als relevante Beschreibungsebenen von Online-Deliberation.

Im Folgenden soll auf Basis empirischer Untersuchungen erörtert werden, inwiefern Twitter ein Medium für den deliberativen Diskurs darstellen kann.

\section{Twitter als „Diskursuniversum“}

Twitter ist nicht nur eine Plattform für die schnelle, synchrone Übermittlung kurzer Nachrichten, sondern lässt sich, wie nachstehend gezeigt werden soll, aufgrund seiner komplexen technischen und kommunikativen Möglichkeiten als ein eigener Diskursraum bezeichnen. Dieser Diskursraum lässt sich durch die twittereigenen Vernetzungsmöglichkeiten auf das gesamte Netz erweitern und kann aus dieser Sicht auch als „Diskursuniversum“ beschrieben werden, da sich Möglichkeiten der kommunikativen, interpersonalen, technischen und thematischen Verknüpfungen von Funktion und Inhalt realisieren lassen (Thimm 2011).

Der Microbloggingdienst Twitter erlaubt es registrierten NutzerInnen, kurze Mitteilungen, sogenannte Tweets, mit maximal 140 Zeichen (Tastenanschläge) auf der Onlineplattform zu veröffentlichen. Beiträge von Twitterusern können abonniert werden und sind dann in der persönlichen Timeline sichtbar. Twitteruser, die Tweets eines Accounts abonnieren, heißen Follower dieses Accounts; die User, denen ein Account folgt, sind dessen Followees.

Die kommunikativ relevanten Elemente auf Twitter, die an anderer Stelle als „Operatorenmodell“ definiert wurden (Thimm/Dang-Anh/Einspänner 2011), beschreiben die zentralen Funktionszusammenhänge im Diskursuniversum Twitter.

\subsection{Das Operatorenmodell}

Die versendeten Mitteilungen auf Twitter (Tweets) können funktional unterschiedlich belegt sein. So sind etwa basale Statusupdates in reiner Textform oder Weiterleitungen von Informationen und Nachrichten ganz anders zu interpretieren als komplexe narrative Strukturen in Tweets, die beispielsweise mit Bild- oder Videomaterial angereichert wurden. Dabei fungieren die Tweets nicht nur als unidirektional gesendete Information eines Users in die Twittersphere, sondern sind in vielen Fällen Bestandteil eines komplexen thematischen Diskurses, der mehrere Teilnehmer, Kontexte und Inhalte miteinander verbindet. 
Diese vielen unterschiedlichen Funktionen werden in Twitter durch die Verwendung spezifischer funktionaler Kommunikationsoperatoren (vgl. Thimm 2011) durch die User realisiert. Funktionale Operationalität bedeutet hierbei, dass die vom User über die Zeichen (@, \#, RT, http://) realisierten Funktionen, wie etwa adressieren, kontextualisieren, zitieren usw. (vgl. Abbildung 1), vom Medium qua technischer Operation realisiert werden. Hierbei ist also jeweils zu unterscheiden zwischen der technischen Operation, die die maschinelle und vernetzte Infrastruktur auf verschiedenen Ebenen (hier: Computer, Internet, Twitter) ausführt, und der kommunikativen Funktion, die zwischen den beteiligten Usern vollzogen wird. Die Bindeglieder zwischen Technik und User sind die Twitter-spezifischen Anweisungen durch digitale Zeichen: die Kommunikationsoperatoren. Durch die Verwendung von Kommunikationsoperatoren lässt man also das technische System bestimmte Operationen durchführen, die eine spezifische kommunikative Funktion haben bzw. erfüllen. Grube bezeichnet diese Art der Verwendung von Zeichen in seiner schriftphilosophischen Auseinandersetzung mit Hypertexten als den „deklarativen Zeichengebrauch“ autooperativer Schriften (Grube 2005: 82).

Die analytische Trennung von Operation und Funktion lässt sich im Handlungsbegriff wieder zusammenführen: In der kommunikativen Handlung, die ein User qua Publikation eines Tweets ausführt, synthetisieren sich diese beiden Teilelemente zu einer Gesamthandlung. Hierbei wird entweder Bezug zu Personen (@, RT), Inhalten (http://) oder Kontexten (\#) genommen. Trotz der Beschränkung auf 140 Zeichen entwickelt sich durch diese verschiedenartigen Kommunikationshandlungen in Twitter also ein hochkomplexes Netzwerk zwischen Personen und den von ihnen publizierten, verlinkten Inhalten zu verschiedenen Themen. Besteht zwischen den einzelnen Beiträgen ein inhaltlich-thematischer Bezug, lässt sich von einem Diskurs sprechen.

Die Möglichkeit, Beiträge entweder durch einen vorangestellten @--Operator oder durch Klicken des „Antworten“-Buttons an bestimmte NutzerInnen zu adressieren bzw. diese in einem Tweet zu erwähnen (Operation), konstituiert einen interpersonalen Diskursraum (Funktion), der prinzipiell öffentlich ist. Durch die Verwendung des \#-Operators wird ein Begriff oder eine Zeichenfolge zum Hashtag, wobei die indexierten Zeichenketten in Twitter automatisch verlinkt werden (Operation). Diese zeichenbasierte Systematisierung ermöglicht eine thematische Kontextualisierung und dadurch eine inhaltsbezogene Diskursorganisation (Funktion). Sowohl bei @-Adressierungen als auch bei Hashtags lassen sich die technisch-operativen von den sprachlich-kommunikativen Elementen trennen: Das erste Zeichen (@, \#) initiiert die (technische) Operation und sorgt somit für die technische Bezugnahme (Verlinkung). Das zweite (sprachliche) Zeichen (Name des Adressaten bzw. Hashtagbenennung) vollzieht hingegen die 
Referenzierung der Person (@) bzw. des Kontextes (\#). Durch die Erstellung von Hyperlinks (http://-Operator), die automatisch verlinkt und gekürzt werden (Operation), lässt sich auf andere Webinhalte verweisen, oder es lassen sich eigene Fotos, Videos oder Geotags einbinden, und somit lässt sich jeweils Bezug zu weiteren Inhalten nehmen (Funktion). Retweeten (RT-Operator) bewirkt, dass man den Tweet eines anderen Autors erneut veröffentlicht. Dies wird durch einen Klick auf den Retweet-Button ausgelöst (Operation) oder manuell initiiert, indem man an den Anfang des wiederholten Tweets „RT @“ und den Namen des Originalabsenders setzt. Wird so ein Beitrag redistribuiert, können nun die eigenen Abonnenten auch den ursprünglichen Tweet lesen, ohne dem ursprünglichen Autor zu folgen. Neben der Diffusion von Tweets dienen Retweets auch der twitterinternen Zitation (vgl. Weller/Dröge/Puschmann 2011) und der Autorenreferenzierung (Funktion).

Das Operatorenmodell zu Twitter lässt sich entsprechend wie folgt abbilden:

\begin{tabular}{|l|l|l|}
\hline Operationale Ebene & & Funktionale Ebene \\
\hline @ (Adressierung, Erwähnung) & $\rightarrow$ & Adressierung \\
& $\rightarrow$ & Interaktion \\
& $\rightarrow$ & Kohärenz \\
& $\rightarrow$ & Kontaktaufnahme \\
\hline \# (Verschlagwortung) & $\rightarrow$ & Themenspezifikation \\
& $\rightarrow$ & Diskursorganisation \\
& $\rightarrow$ & Kontextualisierung \\
\hline http:// (Hyperlinking) & $\rightarrow$ & Argumationsverteilung \\
\hline RT (Redistribution) & $\rightarrow$ & Diffusion \\
& $\rightarrow$ & Referenz \\
\hline
\end{tabular}

Abbildung 1: Operatorenmodell des Diskursuniversums Twitter

Die grundlegende Struktur von Twitter ist von einer Mischung aus Newsmedium, synchroner interpersonaler Kommunikation und sozialer Gruppenbildung bestimmt. Die versandten Mitteilungen auf Twitter können funktional unterschiedlich gerichtet sein. Trotz der Beschränkung auf 140 Zeichen entwickelt sich durch die beschriebenen unterschiedlichen Kommunikationshandlungen in Twitter ein hochkomplexes Bezugnahmesystem zwischen Personen und den von ihnen publizierten, verlinkten Inhalten, die sich in verschiedenen Modalitäten ausdifferenzieren (Bild, Schrift, Ton). 


\subsection{Twitter im deliberativen Diskurs}

Wie bereits erläutert, ist das Kriterium der Zugänglichkeit als Grundbedingung des deliberativen Diskurses für das Internet nicht grundsätzlich gegeben. Diejenigen aber, die im Netz präsent sind, fühlen sich zur Beteiligung und Kritik ermächtigt. Der öffentliche Diskurs wird nicht mehr nur von Medienkonzernen bestimmt, sondern zunehmend auch von einzelnen AktivistInnen. Diese Ermächtigung des Einzelnen stößt zwar durch die zunehmende Kontrolle der großen Medienkonzerne Google, Apple und Facebook an ihre Grenzen, ist jedoch zum aktuellen Zeitpunkt noch als ein Kernstück der Attraktivität des Netzes anzusehen.

Für Menschen, denen der Zugang zum Internet grundsätzlich möglich ist, bleibt auch der Zugang zu Twitter und die Teilnahme an der dort stattfindenden Diskussion nicht verwehrt. Es bedarf lediglich einer E-Mail-Adresse, mit der man sich für das Microbloggingsystem anmelden kann, um anschließend mit einem selbst gewählten Benutzernamen Tweets zu versenden oder zu abonnieren. Twitter erfüllt demnach, abgesehen von den grundsätzlichen Einschränkungen durch die gestiegenen Anforderungen an ,digital literacy“ (Hobbs 2006) und allgemeinen Zugangshindernissen des Digitalen, die grundlegenden Bedingungen und Voraussetzungen, um als Kommunikationsmittel von Digitaler Deliberation gelten zu können (Zugangsfreiheit). Für die Nutzung von Twitter entstehen für die User keine Zusatzkosten.

Kommunikative Deliberationsverfahren in informellen Netzwerken, als welches Twitter klassifiziert wird, unterliegen keinen institutionell vorgegebenen Verfahrensregeln. Jedoch bildet das Kommunikationsmedium, in dem sich deliberative Prozesse abspielen, den Rahmen für die Entfaltungsmöglichkeiten der Deliberation. Twitter ist ein Medium, das ein egalitäres Diskursnetzwerk formiert, in dem sich ein disperses Publikum an unterschiedlichen Diskursen beteiligen kann. Entscheidend für die spezifische Beschaffenheit des Netzwerkes ist die Möglichkeit, Tweets von Usern zu abonnieren, ohne dass es deren Bestätigung bedarf. Dies führt dazu, dass prinzipiell jeder jedem folgen kann. Ebenso sind die Interaktionshürden zwischen der institutionalisierten Politik und der Bürgerschaft gering: Technisch und strukturell macht es keinen Unterschied, ob man einen Tweet an einen Politiker oder an den besten Freund adressiert. Es gibt demnach im technischen Sinne keinen Statusunterschied in der Twitterkommunikation. Die Teilnahmemöglichkeit an (deliberativen) Diskursen ist insofern gewährleistet. Kommunikative Parität ist jedoch nicht zwangsläufig gegeben, da die Verteilung der Aufmerksamkeit in Twitter sehr unausgewogen ist: „Less than $0.05 \%$ of the user population [...] attract almost $50 \%$ of all attention within Twitter", woraus folgt ,information flows have not become egalitarian by any means“ 
(Wu/Hofmann/Mason/Watts 2011: 5). Somit lässt sich konstatieren, dass in Twitter zwar prinzipiell jedem eine Beteiligung am Diskurs möglich ist, jedoch nicht alle Teilnehmer eine Stimme mit gleichem Gewicht haben.

Die Relevanz von Rationalität markiert im Kontext deliberativer Demokratietheorien den Wunsch nach stärkerer Legitimation durch fundierte Argumentation und sich daran anschließender Genese bzw. Ausdifferenzierung politischer Präferenzen. Der damit verbundene normative Anspruch an Diskurse ist jedoch durchaus umstritten (Schaal/Ritzi 2009). Rationale Argumentation in einem demokratischen Prozess bedeutet im Habermas'schen Duktus, dass Beteiligte an einer politischen Diskussion als ,unabdingbare Voraussetzungen diskursiven Handelns" (Brosda 2010: 88) gegenseitig Geltungsansprüche der Wahrheit, Richtigkeit und Wahrhaftigkeit an ihre Äußerungen erheben. „Damit behaupten sie implizit, dass das, was sie sagen, als wahr oder richtig oder wahrhaftig, in jedem Fall als vernünftig akzeptiert werden kann und dass das Gesagte, wenn es problematisiert wird, in diesen Hinsichten rational begründet werden kann“ (Habermas 2008: 149). Mit der Unterstellung von Rationalität ist die Annahme verbunden, dass Aussagen, Stellungnahmen, Argumente und letztlich Entscheidungsbekundungen ehrlich und ohne Täuschungsabsichten zustande kommen.

Problematisch für die Untersuchung von Rationalität in Onlinediskursen (wie auch in Offlinediskursen) ist die Tatsache, dass keine externen Gütekriterien für Argumentationen existieren, zumal das Ergebnis einer Diskussion auf diese Weise normativ vorbestimmt wäre (Bächtiger/Pedrini/Ryser 2010: 198). Es wird daher vorgeschlagen, Begründungsrationalität prozedural zu erfassen, das heißt, die Ausführlichkeit und Ausgestaltung von Gesprächspositionen zu analysieren (ebenda). In Bezug auf Twitter ist hier außerdem an die Messung verwendeter Hyperlinks in den Argumentationen zu denken, die entsprechend dem hier entwickelten Operatorenmodell zur qualitativen Stützung von Aussagen gesetzt werden. Auch die anderen Kommunikationsoperatoren können für eine Argumentationsanalyse von Tweets hilfreich sein. So spielen Retweets bei der Interpretation von rationalen Diskurselementen ebenso eine Rolle wie das Verschlagworten von Argumenten durch Hashtags. Auch das Adressieren bestimmter, bereits im Diskurs involvierter oder auch noch nicht einbezogener Gesprächsteilnehmer ist für das Konstatieren eigener Meinungen und Positionen von sehr großer Relevanz. Neben einer qualitativen Analyse ist es mithin durchaus möglich, Twitterdiskurse auch mit quantitativen Methoden auf Rationalität zu überprüfen.

Information ist eine der basalen Voraussetzungen für Deliberationsprozesse: ,[D]eliberation is debate and discussion aimed at producing reasonable, well-informed opinions [...] in light of discussion, new information, and claims made by fellow participants“ (Chambers 2003: 309). Die Hypertextualität von 
Online-Kommunikationsformen ermöglicht es Usern, Informationen zu erhalten und zu verbreiten. Auf der Achse von Produktion, Distribution und Rezeption ist das distributive Potenzial von Twitter zur Verlinkung von Webadressen durch den http://-Operator (Thimm et al. 2011: 275ff.) entscheidend. Im Zusammenhang damit stehen die Produktionsbedingungen, die es erfordern, den http://-Operator zweckgemäß einzusetzen und den nicht-linearen Strukturen von Tweets als Hypertexten anzupassen.

Twitter-Nutzer können also, bedingt durch das technisch-funktionale Distributionsvermögen des Mediums, Links zu anderen Inhalten setzen. Hierbei werden multimodale Inhalte (Videos, Fotos, Slideshows etc.) einiger Anbieter wie etwa YouTube oder Flickr innerhalb von Twitter in der Detailansicht auf der rechten Seite dargestellt. Relevant für die Verteilung von Informationen sind jedoch vor allem Links auf Seiten, die Informationen schriftlich präsentieren, wie etwa Webseiten von Nachrichtenportalen und Blogs. Die verlinkten Inhalte dienen hierbei zum einen dem grundsätzlichen Verteilen von Information, zum anderen, in einer deliberativen Argumentationssequenz, ebenso dem Stützen von Argumenten: „[D]ie Bedeutung des Tweets [erhöht sich, d. V.] durch die Verlinkung und erlangt somit einen höheren argumentativen Wert im Diskurs" (ebenda: 276).

Die korrekte und zielführende Produktion von Hyperlinks obliegt hierbei stets der Produktionskompetenz der User. Die zur Verfügung stehenden 140 Zeichen erfordern eine zeichenökonomische Ausgestaltung von Tweets. Hyperlinks, die mitunter auf lange URLs verweisen, werden automatisch verkürzt. Rezeptionskompetenz bedeutet auch, Inhalte nach Relevanz zu selektieren und Informationen dementsprechend im Diskurs zu verorten. Zudem muss der Rezipient nach der „transmedialen Grenzüberschreitung“ (Wirth 2006: 22) einen Weg zurück von reinen Rezeptionsmedien in das Kommunikationsmedium Twitter finden.

Eine Facette des Deliberationspotenzials von Twitter offenbart sich also in den Distributionsmöglichkeiten, die die Microblogging-Plattform durch die automatische Verlinkung (http://-Operator) bereitstellt. Auf Seiten der Produktion und Rezeption hingegen sind es individuelle Nutzerentscheidungen und -verhaltensweisen, die ihre Gestaltung und Ausdifferenzierung prägen. 


\section{Twitternutzung im politischen Diskurs: Exemplarische Analysen}

Das Operatorenmodell erfasst nicht nur die wichtigsten kommunikativen Funktionalitäten innerhalb des Microbloggingsystems Twitter, sondern dient auch der Operationalisierung empirischer Forschungsfragen. Die nachstehend analysierten Tweets sind im Kontext von Landtagswahlen erhoben worden, von Politikern oder interessierten BürgerInnen verfasst und beschäftigen sich mit Politik. Somit bilden sie eine adäquate Grundlage für die Analyse deliberativer Prozesse auf Twitter.

Zugrunde liegt ein Datensatz von 20.414 Tweets, die in einem Zeitraum von je vier Wochen (drei Wochen vor und einer Woche nach dem jeweiligen Wahltermin) zu den Landtagswahlen 2011 in Sachsen-Anhalt, Baden-Württemberg und Rheinland-Pfalz erhoben wurden. Sowohl die Datenerhebung (per RSS-FeedReader) als auch die anschließende Auswertung (per QDA-Software) erfolgten in computergestützten Verfahren. Einbezogen wurden dabei jeweils die vier twitteraktivsten KandidatenInnen einer Partei, der jeweilige Landes-Account der Parteien sowie die Tweets der interagierenden Bürgerinnen und Bürger. Zur Aufzeichnung dieser Tweets wurden für den Untersuchungszeitraum standardisierte Suchvorgänge mit der Twitter-internen Suchmaschine durchgeführt. Grundlage waren jeweils die Twitter-spezifischen Adressierungen an die politischen Accounts sowie die Auswahl der Tweets nach Hashtags, die im Zusammenhang mit den Landtagswahlen standen (z. B. \#ltw, \#ltw11, \#ltwsa, \#ltwbawü, \#ltwrlp u. v. m.). Tweets, die den hier exemplifizierten Interaktionssequenzen zugehörig sind, jedoch nicht den Erhebungskriterien entsprachen, wurden manuell ergänzt. Auf diese Weise wurden die öffentlich stattfindenden Interaktionen zwischen den untersuchten Accounts extrahiert und archiviert. Das hier fokussierte Untersuchungsinteresse richtete sich insbesondere auf die Interaktion zwischen den am Twitterdiskurs partizipierenden Bürgerinnen und Bürgern.

Folgende beispielhafte Interaktion zeigt, wie Twitter im Landtagswahlkampf eingesetzt wird: 


\begin{tabular}{|c|c|c|}
\hline Twittername & Zeit & Tweet \\
\hline occi00 & $\begin{array}{l}28.02 .2011 \\
11: 10\end{array}$ & $\begin{array}{l}\text { Das grüne Team für Sachsen-Anhalts Landtag heute in } \\
\text { der \#Volksstimme: http://bit.ly/eb7/9o \#fb \#ltwlsa \#zweit- } \\
\text { stimmegruen }\end{array}$ \\
\hline cyberfux & $\begin{array}{l}28.02 .2011 \\
11: 10\end{array}$ & $\begin{array}{l}\text { @occi00 dieses jahr kann man echt niemanden wählen } \\
\text {;-) \#ltwsa }\end{array}$ \\
\hline occio0 & $\begin{array}{l}28.02 .2011 \\
12: 14\end{array}$ & @cyberfux Das sehe ich naturgemäß anders ;-) \\
\hline cyberfux & $\begin{array}{l}28.02 .2011 \\
12: 14\end{array}$ & $\begin{array}{l}\text { @occi00 logischerweise ;-) aber ich bin ja durchaus ein- } \\
\text { sichtig: überzeug mir ;-) }\end{array}$ \\
\hline occio0 & $\begin{array}{l}28.02 .2011 \\
13: 06\end{array}$ & $\begin{array}{l}\text { @cyberfux hier gibts Gute Gründe für Grün: http://tinyurl. } \\
\text { com/4gedbpy }\end{array}$ \\
\hline
\end{tabular}

Abbildung 2: Beispiel für Informationsverteilung durch Hyperlinks auf Twitter

Twitteruser Sebastian Lüdecke alias „occi00“ trat bei der Landtagswahl in Sachsen-Anhalt im März 2011 als Direktkandidat von Bündnis 90/Die Grünen an. In der gezeigten Interaktion verweist er zunächst auf einen Zeitungsartikel. Sein initialer Tweet ist mit Hashtags gekennzeichnet und beinhaltet einen Shortlink, der auf eine Seite der regionalen sächsischen Tageszeitung „Volksstimme“ führt. Der User „,cyberfux“ adressiert Lüdecke nun per @-Operator. Hieraus entwickelt sich eine kurze Interaktion, im Zuge derer cyberfux Lüdecke auffordert, ihn von sich bzw. seiner Partei zu überzeugen. Im letzten abgebildeten Tweet verweist Lüdecke nun per Shortlink auf eine parteieigene Seite, auf der kurz und knapp zehn Punkte des politischen Programms von Bündnis 90/Grüne in Sachsen-Anhalt aufgelistet sind.

Das umfangreiche Informationspotenzial der Hyperlinks in Twitter wird hier ebenso ersichtlich wie die Grenzen, die Tweets durch die Beschränkung auf 140 Zeichen für die Kommunikatoren beinhalten. In argumentativen Zusammenhängen greifen die User häufig auf externe Quellen in weiteren Medien zurück. Der http://-Operator verlinkt hierbei automatisch Zeichenketten, die mit „http://“ beginnen und verkürzt sie gegebenenfalls. Inhalte werden schnell verfügbar gemacht. Die Shortlinks sorgen zudem dafür, dass die Inhalte, die sich hinter dem Link verbergen, teilweise nicht ohne Klick bekannt sind. Kontaktaufnahme, Kohärenz und Interaktion werden so durch den@-Operator generiert, Informationsverteilung und Argumentation hingegen durch den Hypertextualität erzeugenden http://-Operator. 
Mit dem oben eingeführten weiten Begriff von deliberativen Kommunikationsprozessen stellt sich die Frage, wie sich Dissens in politischer Kommunikation in Twitter sprachlich ausdifferenziert. Grundlegend für die Durchführung deliberativer Prozesse ist, neben der inhaltlichen Qualität der Argumente, die Kompetenz der Diskursteilnehmer, sich aufeinander so zu beziehen, dass Argumentstrukturen sichtbar werden. Insofern sind nicht nur die geäußerten Inhalte, sondern auch die durch das Medium Twitter bereitgestellten Kommunikationsoptionen (Operationen) für das Gelingen deliberativer Verfahren essentiell.

Im folgenden Beispiel handelt es sich, ebenso wie in der oben analysierten Sequenz, um eine durchweg per @-Operator ausgeführte Interaktion. Initiativ ist hierbei ein Retweet des Parteien-Accounts der Landes-FDP in Sachsen-Anhalt (fdp_lsa), der im Original von dem User „KrassPeter“ (im Folgenden abgekürzt: „K.“) stammt. Auf diesen Tweet wiederum reagiert der User „NeoXtrim“ (,N.“) - offenbar ein Sympathisant der Piratenpartei - und startet im Folgenden eine Diskussion mit dem Urheber des Tweets.

\begin{tabular}{|l|l|l|l|}
\hline & Twittername & Zeit & Tweet \\
\hline 1 & fdp_Isa & $\begin{array}{l}21.03 .2011 \\
11: 58\end{array}$ & $\begin{array}{l}\text { RT @KrassPeter: An die Erben von \#Genscher } \\
\text { in Halle: Anlauf holen! \#Grüne sind ganz klar eine } \\
\text { \#Brückentechnologie. @liberal_2011 @FDP_LSA } \\
\text { \#FDP \#ltwsa \#lsa }\end{array}$ \\
\hline 2 & NeoXtrim & $\begin{array}{l}21.03 .2011 \\
12: 00\end{array}$ & $\begin{array}{l}\text { Und ihr bereits ausgesondert. RT @KrassPeter: } \\
\text { \#Güne sind ganz klar eine \#Brückentechnologie. @ } \\
\text { liberal_2011 @FDP_LSA \#ltwsa }\end{array}$ \\
\hline 3 & KrassPeter & $\begin{array}{l}21.03 .2011 \\
12: 36\end{array}$ & $\begin{array}{l}\text { @NeoXtrim sprach der \#Pirat über die regierende } \\
\text { Partei :-). }\end{array}$ \\
\hline 4 & NeoXtrim & $\begin{array}{l}21.03 .2011 \\
12: 57\end{array}$ & $\begin{array}{l}\text { @KrassPeter jo :-) oder willst du allen ernstes be- } \\
\text { haupten, dass 3,8\% für FDP (seit 1990 in LSA) vs. } \\
\text { 1,4\% Piraten (erster Antritt) gut ist? }\end{array}$ \\
\hline 5 & KrassPeter & $\begin{array}{l}21.03 .2011 \\
13: 24\end{array}$ & $\begin{array}{l}\text { @NeoXtrim Verehrter orangefarbener Freund, die } \\
\text { \#ltwsa ist nicht zu vergleichen mit der \#Bundestags- } \\
\text { wahl \& der \#ltwbw. Beweis: \#NPD \& \#Linke. }\end{array}$ \\
\hline 6 & KrassPeter & $\begin{array}{l}21.03 .2011 \\
13: 31\end{array}$ & $\begin{array}{l}\text { @NeoXtrim Wie kommt es eigentlich, dass ihr uns } \\
\text { nicht mögt, wo wir doch den Bürgerrechts-Ansatz } \\
\text { gemeinsam haben? Abgrenzungsbedürfnis? }\end{array}$ \\
\hline 7 & NeoXtrim & $\begin{array}{l}21.03 .2011 \\
13: 50\end{array}$ & $\begin{array}{l}\text { @KrassPeter Lobbyismus, VDS jetzt nennt ihr es } \\
\text { Quick Freeze), Atompolitik, inr tut nichts gegen Ar- } \\
\text { mut, seid für Studiengebühren. mehr? }\end{array}$ \\
\hline
\end{tabular}




\begin{tabular}{|c|c|c|c|}
\hline 8 & KrassPeter & $\begin{array}{l}21.03 .2011 \\
14: 02\end{array}$ & $\begin{array}{l}\text { @NeoXtrim Lobbyismus ist meiner Auffassung nach } \\
\text { ein Problem, alles andere ist Quatsch. Trotzdem } \\
\text { danke, jetzt hab ich einen Eindruck. }\end{array}$ \\
\hline 9 & NeoXtrim & $\begin{array}{l}21.03 .2011 \\
14: 03\end{array}$ & $\begin{array}{l}\text { @KrassPeter alles andere ist Quatsch? Danke, jetzt } \\
\text { habe ich ebenso einen Eindruck. }\end{array}$ \\
\hline 10 & KrassPeter & $\begin{array}{l}21.03 .2011 \\
14: 10\end{array}$ & $\begin{array}{l}\text { @NeoXtrim Die \#FDP für nachgelagerte } \\
\text { Studiengeb.,damit nicht die arbeitende Bevölkerung } \\
\text { den späteren Besserverdienern die Ausbildung be- } \\
\text { zahlt }\end{array}$ \\
\hline 11 & KrassPeter & $\begin{array}{l}21.03 .2011 \\
14: 11\end{array}$ & $\begin{array}{l}\text { @NeoXtrim Und im Übrigen ist sie natürlich auch ge- } \\
\text { gen VDS. Von daher: ja, bisweilen war das Quatsch. }\end{array}$ \\
\hline 12 & NeoXtrim & $\begin{array}{l}21.03 .2011 \\
14: 24\end{array}$ & $\begin{array}{l}\text { @KrassPeter ach, komisch, dass StudisOnline das } \\
\text { anders sieht: http://bit.ly/hzbh2r - zu VDS: http://bit. } \\
\text { ly/gVy4nr }\end{array}$ \\
\hline 13 & KrassPeter & $\begin{array}{l}21.03 .2011 \\
14: 36\end{array}$ & $\begin{array}{l}\text { @NeoXtrim Nur ein Pirat würde die Interessen der } \\
\text { Strafverfolgung in Gänze außer Acht lassen ;-). Zu } \\
\text { Studiengebühren: Was studierst du? }\end{array}$ \\
\hline 14 & NeoXtrim & $\begin{array}{l}21.03 .2011 \\
14: 42\end{array}$ & $\begin{array}{l}\text { @KrassPeter sicher nicht, siehe: http://bit.ly/emo- } \\
\text { gYV - was tut es zur sache was und ob ich studiere? }\end{array}$ \\
\hline 15 & KrassPeter & $\begin{array}{l}21.03 .2011 \\
14: 53\end{array}$ & $\begin{array}{l}@ \text { NeoXtrim \#Liberalismus ist nicht so einfach zu } \\
\text { verstehen.Deshalb hilft es,sich seine eigene Situati- } \\
\text { on zu vergegenwärtigen.Nur ein Vorschlag }\end{array}$ \\
\hline 16 & NeoXtrim & $\begin{array}{l}21.03 .2011 \\
14: 55\end{array}$ & $\begin{array}{l}\text { @KrassPeter Du liest nicht einmal oder? Typisch. } \\
\text { Warum sollte es auch was bringen sich zu informie- } \\
\text { ren. Du willst nur hören was du eh denkst }\end{array}$ \\
\hline 17 & KrassPeter & $\begin{array}{l}21.03 .2011 \\
15: 11\end{array}$ & $\begin{array}{l}@ \text { NeoXtrim Die meisten Liberalen haben einen Job } \\
\text { und somit Montag Nachmittag keine Zeit, ad hoc sei- } \\
\text { tenweise zu lesen.Ich komm auf dich zurück }\end{array}$ \\
\hline
\end{tabular}

Abbildung 3: Twitterinteraktion FDP-Sympathisant und Piraten-Sympathisant

Bemerkenswert ist hierbei zunächst, dass die beiden Diskutanten über den initiativen (Re-)Tweet eines Dritten (fdp_lsa) aufeinander aufmerksam werden. Hierbei offenbart sich das Diffusionspotenzial von Retweets: Inhalte von Usern werden weiterverteilt und erreichen somit auch User, die die Tweets des Ursprungsautors möglicherweise in ihrer Timeline nicht empfangen hätten. $\mathrm{Zu}$ der Konstellation wie sie hier vorliegt - die Interaktion zweier Sympathisanten unterschiedlicher Parteien - war es erforderlich, dass bei beiden eine Verbindung zum Retweet-Account, als Follower (,NeoXTrim") oder Followee („KrassPeter"), bestand. Das bedeutet also für das aufgeführte Beispiel, dass der Piraten-Sympathisant dem 
FDP-Partei-Account folgte, um den Tweet in seiner Timeline lesen zu können oder über eine Suchanfrage bzw. einen Hashtag auf ihn aufmerksam wurde. Die Rezeption erfolgt auf Grundlage der Selektion des Followers. Auf Seiten der Produktion bedeutet das wiederum, dass Tweets im Rahmen politischer Kommunikation, soweit sie strategisch ausgerichtet ist, wie etwa bei einem Partei-Account, mehrfach adressiert sein können.

Auffällig ungleich verteilt ist die Verwendung von Hashtags: Während K. in vier von zehn Tweets Hashtags benutzt $(3,5,10,15)$, verzichtet $N$. bis auf in Retweets zitierte Hashtags (2) vollkommen auf deren Verwendung. Hyperlinks hingegen werden ausschließlich von $\mathrm{N}$. verwendet $(12,14)$ und erlangen im Laufe der Diskussion argumentative Bedeutung.

Die Diskussion, die sich in der Beispielsequenz entwickelt, nimmt ab Tweet 6 eine Eigendynamik und spaltet sich thematisch von der vorgehenden, sich auf den Eingangstweet beziehenden, ab. K. fragt N. nach Gründen für die Ablehnung der FDP durch Piraten-Sympathisanten (6), also nach den Unterschieden zwischen den Parteien. N.s Meinung nach gibt es unterschiedliche Auffassungen bzw. Probleme in den Themenbereichen: Lobbyismus, Vorratsdatenspeicherung, Atompolitik, Sozialpolitik und Studiengebühren (7). Bis auf den ersten Punkt wiegelt K. die Vorwürfe ab (,,alles andere ist Quatsch“, 8) und bringt im Weiteren auf Nachfrage (9) Argumente gegen N.'s Auffassung (10) bzw. widerspricht seiner Auffassung (11). In (12) nun verwendet N. erstmals den http://-Operator, um auf externe Quellen zu verweisen, die wiederum K.'s Behauptungen widersprechen. So weist der erste Link in (12) auf einen Artikel des Portals „Studis online“ mit dem Titel „Auch nachgelagerte Studiengebühren sind Studiengebühren“. Der zweite Link referiert auf einen Eintrag im Blog ,moenikes.de“ mit dem Titel „Quick Freeze - der Wolf im Schafspelz“, der sich kritisch mit dem Konzept „Quick Freeze“ (eine Alternative zum Konzept der Vorratsdatenspeicherung) auseinandersetzt. Im weiteren Verlauf wiederholt sich dieses Muster: Argument von K., Gegenargument von N. per Hyperlink. Letztlich verweist K. darauf, dass er keine Zeit habe, die verlinkten Seiten zu diesem Zeitpunkt zu lesen und vertagt die Diskussion (,Ich komm auf dich zurück“, 17).

Das Beispiel zeigt, dass Hyperlinks vor allem dafür eingesetzt werden, um auf Inhalte zu verweisen, mit denen die Argumentation gestützt wird. Realisiert wird das durch Bezugnahme auf den Adressaten qua @-Operator sowie durch Verlinkung durch den http://-Operator. 


\section{$5 \quad$ Fazit}

In Twitter formiert sich ein eigenständiger Diskursraum (,Diskursuniversum“), der durch die funktionale Aufladung der vier twitterspezifischen Zeichen (RT, http, @,\#) zu Operatoren eine eigene Form der Diffusion, Information, interpersonalen Interaktion und Diskurstrukturierung ermöglicht. Um Twitter diskursiv nutzen zu können, müssen die User diese Twitter-spezifischen Optionen ziel- und zweckgerichtet einsetzen und sinnvoll mit sprachlichen Äußerungen im Diskurs verknüpfen. Wie sich bei entsprechender Umsetzung hoch komplexe Diskurse gestalten lassen, wurde an dem dargestellten Dialog ersichtlich.

Während sich deliberative Kommunikationsprozesse in Twitter also strukturell durchaus entfalten können, sind auf der inhaltlichen Seite sowohl Möglichkeiten eröffnet, in Form von Verlinkungen durch den http://-Operator, als auch Grenzen gesetzt. Die Kürze der Tweets und die daraus resultierende Schnelligkeit des Mediums charakterisieren Twitter eher als Informationsverteilungs-, denn als Diskussionsplattform. Dies zeigen auch die aktuellen Studien zur Frage des Einflusses von Twitter im Kontext des „Arabischen Frühlings“ (Christensen 2011).

Auf der empirischen Ebene ist die Untersuchung der Mediatisierung des Politischen durch die Internetkommunikation im Sinne deliberativer Strukturen sowohl hinsichtlich der methodischen als auch im Bezug auf die interpretative Vorgehensweise als diffizil anzusehen. Die These, dass das Internet zu einer deliberativeren Diskurskultur führen kann, in der Politik und Zivilgesellschaft egalitär diskutieren, lässt sich nur in ersten Ansätzen belegen. Wenngleich sich digitale Medien und Onlinekommunikation aus politischen Kampagnen heute nicht mehr wegdenken lassen, ist für eine makroperspektivische Aussage eine Langzeitstudie erforderlich.

Es bleibt jedoch zu fragen, welche Funktion der Online-Distribution von Informationen, die in Sekundenschnelle jegliche geografische Grenze überwinden kann, im Zusammenhang mit deliberativer Demokratie zugesprochen werden kann. Wurde bisher der Problematik des ,informierten Bürgers“ nur begrenzt Aufmerksamkeit geschenkt und eher über dessen Unwilligkeit sich zu informieren geklagt, so erscheint dies spätestens seit den intensiven Diskussionen um Wikileaks in einem anderen Licht. Sieht man den Zugang zu Informationen als eine zentrale Grundlage für deliberative Diskurse an, so erhält diese Funktion von Twitter als Distributionsmedium eine neue Bedeutung. Die Möglichkeit, auch gegen den Willen von Regierungen eigenständige Informationskanäle zur Partizipation zu öffnen und so die Option zur Diskussion zu ermöglichen, muss als ein wichtiger Baustein eines Modells zur Online-Deliberation angesehen werden. 


\section{Literatur}

Bächtiger, André/Pedrini, Seraina/Ryser, Mirjam (2010): Prozessanalyse politischer Entscheidungen: Deliberative Standards, Diskurstypen und Sequenzialisierung. In: Behnke, Joachim/Bräuninger, Thomas/Shikano, Susumu (Hrsg.): Jahrbuch für Handlungs- und Entscheidungstheoric. Band 6: Schwerpunkt Neuere Entwicklungen des Konzepts der Rationalität und ihre Anwendungen. Wiesbaden: VS Verlag, S. 193-226.

Bessette, Joseph/Pitney, John (2011): American Government and Politics: Deliberation, Democracy and Citizenship, Election Update. Boston: Wadsworth Publishing.

Boyd, Danah (2008): Can Social Network Sites Enable Political Action? In: International Journal of Media and Cultural Politics, 4 (2), S. 241-244.

Brosda, Carsten (2010): Diskursethik. In: Schicha, Christian/Brosda, Carsten (Hrsg.): Handbuch Medienethik. Wiesbaden: VS Verlag, S. 83-106.

Chambers, Simone (2003): Deliberative Democracy Theory. In: Annual Review Political Science, 6, S. 307-326.

Christensen, Christian (2011): Twitter Revolutions? Addressing Social Media and Dissent. In: The Communication Review, 14 (3), S. 155-157.

Döring, Nicola (2008): Reduced Social Cues / Cues Filtered Out Approach. In: Krämer, Nicole/ Schwan, Stephan/Unz, Dagmar (Hrsg.): Medienpsychologie. Schlüsselbegriffe und Konzepte. Stuttgart: Kohlhammer, S. 290-297.

Dyson, Esther (1997): Release 2.0. A Design for Living in the Digital Age. New York: Broadway.

Fishkin, James S. (1991): Democracy and Deliberation. New Directions for Democratic Reform. New Haven, London: Yale University Press.

Fishkin, James S. (2009): Dissensus on Consensus. In: http://www.cato-unbound.org/2009/02/24/ james-fishkin/dissensus-on-consensus/ [27.10.2011].

Grube, Gernot (2005): Autooperative Schrift - und eine Kritik der Hypertexttheorie. In: Sybille Krämer (Hrsg.): Schrift. Kulturtechnik zwischen Auge, Hand und Maschine. München: Fink, S. 81-114.

Grunwald, Armin/Banse, Gerhard/Coenen, Christopher/Hennen, Leonhard (2006): Netzöffentlichkeit und digitale Demokratie: Tendenzen politischer Kommunikation im Internet. (Studien des Büros für Technikfolgen-Abschätzung beim Deutschen Bundestag, 18). Berlin: Edition Sigma.

Haas Lyons, Susanna (2011): Facebook as a Site of Public Deliberation on Local Sustainability Policy. Paper zur ACM CHI Conference on Human Factors in Computing Systems 2011, Vancouver. In: http://staccyk.org/chil1 workshop/submissions/HaasLyons.pdf [27.10.2011].

Habermas, Jürgen (1962/1990): Strukturwandel der Öffentlichkeit. Untersuchungen zu einer Kategorie der bürgerlichen Gesellschaft. Frankfurt a. M.: Suhrkamp.

Habermas, Jürgen (1983): Moralbewusstsein und kommunikatives Handeln. Frankfurt a. M.: Suhrkamp.

Habermas, Jürgen (1996): Drei normative Modelle der Demokratie. In: Habermas, Jürgen: Die Einbeziehung des Anderen. Studien zur politischen Theorie. Frankfurt a. M.: Suhrkamp, S. 277-292.

Habermas, Jürgen (2008): Ach, Europa. Frankfurt a. M.: Suhrkamp.

Heacock, Rebekah (2009): Twitter Revolution? In: http://jackfruity.com/wp-content/uploads/2009/12/ Heacock TwitterRevolution.pdf [27.10.2011].

Hobbs, Renee (2006): Multiple visions of multimedia literacy: Emerging areas of synthesis. In: McKenna, Michael/Labbo, Linda/Kieffer, Ron/Reinking David (Hrsg.): Handbook of Literacy 
and Technology, Volume II. International Reading Association. Mahwah: Lawrence Erlbaum Associates, S. 15-28.

Idle, Nadia/Nunns, Alex (2011) (Hrsg.): Tweets from Tahrir. Egypt's revolution as it unfolded, in the words of the people who made it. New York: OR Books.

Keen, Andrew (2007): The cult of the amateur: how today's internet is killing our culture. New York u. a.: Doubleday.

Kiesler, Sara/Siegel, Jane/McGuire, Timothy (1984): Social Psychological Aspects of Computer-Mediated Communication. In: American Psychologist, 39 (10), S. 1123-1134.

Koop, Royce/Jansen, Harold (2009): Political Blogs and Blogrolls in Canada: Forums for Democratic Deliberation? In: Social Science Computer Review, 27 (2), S. 155-173.

Krotz, Friedrich (2007): Mediatisicrung: Fallstudien zum Wandel von Kommunikation. Wiesbaden: VS Verlag.

Lanier, Jaron (2006): Digital Maoism: The Hazards of the New Online Collectivism. In: http://www. edgc.org/3rd_culture/lanicr06/lanier06_index.html [27.10.2011].

Lévy, Picrre (1998): Cyberkultur. In: Bollmann, Stefan/Heibach, Christiane (Hrsg.): Kursbuch Internet. Anschlüsse an Wirtschaft und Politik, Wissenschaft und Kultur. Mannheim: Rowohlt, S. 56-82.

Lévy, Pierre (2001): Internet und Sinnkrise. In: Maresch, Rudolf/Rötzer, Florian (Hrsg): Cyberhypes. Möglichkeiten und Grenzen des Internet. Frankfurt: Suhrkamp, S. 233-248.

Marr, Mirko/Zillien, Nicole (2010): Digitale Spaltung. In: Schweiger, Wolfgang/Beck, Klaus (Hrsg.): Handbuch Onlinekommunikation. Wiesbaden: VS Verlag, S. 257-282.

Min, Seong-Jac (2007): Online vs. Face-to-Face Deliberation: Effects on Civic Engagement. In: Journal of Computer-Mediated Communication, 12 (4), art. 11.

Morozov, Evgeny (2009): Iran: Downside to the „Twitter Revolution“. In: Dissent, 56 (4), S. 10-14, http://muse.jhu.edu/content/crossref/journals/dissent/v056/56.4.morozov.html [27.10.2011].

Mutz, Diana (2002): Cross-Cutting Social Networks: Testing Democratic Theory in Practice. In: American Political Science Review, 96 (1), S. 111-126.

Negroponte, Nicholas (1995): Being Digital. New York: Random House.

Norris, Pippa (2001): Digital Divide: Civic Engagement, Information Poverty and the Internet Worldwide. Cambridge: Cambridge University Press.

Perlot, Flooh (2008): Deliberative Demokratie und Internetforen - Nur eine virtuelle Demokratie? Baden-Baden: Nomos.

Petrik, Klaus (2010): Deliberation and Collaboration in the Policy Process: A Web 2.0 approach. In: eJournal of eDemocracy and Open Government 2 (1), S. 18-27.

Plake, Klaus/Jansen, Daniel/Schuhmacher, Birgit (2001): Öffentlichkeit und Gegenöffentlichkeit im Internet: politische Potenziale der Medienentwicklung. Wiesbaden: Westdeutscher Verlag.

Rheingold, Howard (1993): Virtual Community. Homesteading on the Electronic Frontier. Reading: Addison-Wesley.

Schaal, Gary S./Ritzi, Claudia (2009): Empirische Deliberationsforschung. MPIfG Working Paper 09/9. In: http://www.mpifg.de/pu/workpap/wp09-9.pdf [27.10.2011].

Schulz, Winfried (2011): Politische Kommunikation. Theoretische Ansätze und Ergebnisse empirischer Forschung. 3., überarbeitete Auflage. Wiesbaden: VS Verlag.

Shirky, Clay (2009): Q\&A with Clay Shirky on Twitter and Iran. In: TED Blog, http://blog.ted. com/2009/06/16/qa_with_clay_sh/[27.10.2011].

Shirky, Clay (2011): The Political Power of Social Media. Technology, the Public Sphere, and Political Change. In: Foreign Affairs, Jan/Feb 2011., http://www.gpia.info/files/u1392/Shirky_Political_Poewr_of_Social_Media.pdf[27.10.2011]. 
Sunstein, Cass (2001): Republic.com. Princeton: University Press.

Surowiecki, James (2004): The wisdom of crowds: Why the many are smarter than the few and how collective wisdom shapes business, economies, societies, and nations. New York, u. a.: Doubleday.

Thierer, Adam (2010): The Case for Internet Optimism, Part 1: Saving the Net from Its Detractors. In: Szoka, Berin/Marcus, Adam (Hrsg.): The Next Digital Decade. Essays on the Future of the Internet. Washington, D.C.: TechFreedom, S. 57-87.

Thimm, Caja (2008): Technically-mediated interpersonal communication. In: Antos,Gerd/Ventola, Eija/Weber, Tilo (Hrsg.): Handbook of interpersonal communication. Berlin, New York: Mouton de Gruyter, S. 331-353.

Thimm, Caja (2011): Ökosystem Internet - Zur Theorie digitaler Sozialität. In: Anastasiadis, Mario/ Thimm, Caja (2011) (Hrsg.): Social Media - Theorie und Praxis digitaler Sozialität. Frankfurt a. M.: Peter Lang, S. 21-42.

Thimm, Caja/Dang-Anh, Mark/Einspänner, Jessica (2011): Diskurssystem Twitter: Semiotische und handlungstheoretische Perspektiven. In: Anastasiadis, Mario/Thimm, Caja (2011) (Hrsg.): Social Media - Theoric und Praxis digitaler Sozialität. Frankfurt a. M.: Peter Lang, S. 265-286.

Thurlow, Crispin/Lengel, Linda/Tomic, Anthony (2004): Computer Mediated Communication, Social Interaction and the Internet. London: Sage.

Vowe, Gerhard (2006): Mediatisierung der Politik? Ein theoretischer Ansatz auf dem Prüfstand. In: Publizistik, 4 (51), S. 437-455.

Weller, Katrin/Dröge, Evelyn/Puschmann, Cornelius (2011): Citation Analysis in Twitter: Approaches for Defining and Measuring Information Flows within Tweets during Scientific Conferences. In: Rowe, Matthew/Stankovic, Milan/Dadzie, Aba-Sah/Hardey, Mariann (Hrsg.): Making Sense of Microposts, Proceedings of the ESWC2011 Workshop on ,Making Sense of Microposts": Big things come in small packages (CEUR Workshop Proceedings, Vol. 718), http://ceur-ws. org/Vol-718/msm2011_proceedings.pdf [27.10.2011], S. 1-12.

Winkel, Olaf (2001): Die Kontroverse um die demokratischen Potenziale der interaktiven Informationstechnologien - Positionen und Perspektiven. In: Publizistik, 2, S. 140-161.

Wirth, Uwe (2006): Hypertextuelle Aufpfropfung als Übergangsform zwischen Intermedialität und Transmedialität. In: Meyer, Urs/Simanowski, Roberto/Zeller, Christoph (Hrsg.): Transmedialität. Zur Ästhetik paraliterarischer Verfahren. Göttingen: Wallstein, S. 19-38.

Witschge, Tamara (2004): Online Deliberation: Possibilities of the Internet for Deliberative Democracy. In: Shane, Peter M. (Hrsg.): Democracy online. New York: Routledge, S. 109-122.

Wu, Shaomei/Hofman, Jake M./Mason, Winter A./Watts, Duncan J. (2011): Who Says What to Whom on Twitter. In: http://research.yahoo.com/files/twitter-flow.pdf [27.10.2011].

Yang, Mundo (2008): Jenseits des „Entweder-Oder“ - Internet als konventioneller Teil der Demokratic. In: kommunikation@gesellschaft, Jg. 9, Beitrag 3, http://www.soz.uni-frankfurt.de/K.G/ B3_2008_Yang.pdf [27.10.2011]. 